\title{
Determining Knots with Quadratic Polynomial Precision
}

\author{
Zhang Caiming ${ }^{1,2}$, Ji Xiuhua $^{1}$, and Liu Hui ${ }^{1}$ \\ ${ }^{1}$ School of Computer Science and Technology, University of Shandong Economics, \\ Jinan 250014, China \\ ${ }^{2}$ School of Computer Science and Technology, Shandong University, \\ Jinan 250061, China
}

\begin{abstract}
A new method for determining knots in parametric curve interpolation is presented. The determined knots have a quadratic polynomial precision in the sense that an interpolation scheme which reproduces quadratic polynomials would reproduce parametric quadratic polynomials if the new method is used to determine knots in the interpolation process. Testing results on the efficiency of the new method are also included.
\end{abstract}

Keywords: parametric curves, knots, polynomials.

\section{Introduction}

The problem of constructing parametric interpolating curves is of fundamental importance in CAGD, CG, scientific computing and so on. The constructed curve is often required to have a better approximation precision and as well as to have the shape suggested by the data points.

The construction of an ideal parametric interpolating curve requires not only a good interpolation method, but also appropriate choice of the parameter knots. In parametric curve construction, the chord length parametrization is a widely accepted and used method to determine knots [1] 2]. Other two useful methods are centripetal model[3] and adjusted chord length method ([4, referred as Foley's method). When these three methods are used, the constructed interpolant can only reproduce straight lines. In paper [5], a new method for determining knots is presented (referred as ZCM method). The knots are determined using a global method. The determined knots can be used to construct interpolants which reproduce parametric quadratic curves if the interpolation scheme reproduces quadratic polynomials.

A new method for determining knots is presented in this paper. The knots associated with the points are computed by a local method. The determined knots have a quadratic polynomial precision. Experiments showed that the curves constructed using the knots by the new method generally has the better interpolation precision.

The remaining part of the paper is arranged as follows. The basic idea of the new method is described in Section 2. The choice of knots by constructing a 
parametric quadratic interpolant to four data points is discussed in Section 3 . The comparison of the new method with other four methods is performed in Section 4. The Conclusions and Future Works is given in Section 5 .

\section{Basic Idea}

Let $P_{i}=\left(x_{i}, y_{i}\right), 1 \leq i \leq n$, be a given set of distinct data points which satisfies the condition that for any point $P_{i}, 1<i<n$, there are at least two sets of four consecutive convex data points, which include it. As an example, for the data points in Figure 3 the point $P_{i+1}$ belongs the two sets of consecutive convex data points which are $\left\{P_{i-2}, P_{i-1}, P_{i}, P_{i+1}\right\}$ and $\left\{P_{i}, P_{i+1}, P_{i+2}, P_{i+3}\right\}$, respectively. The goal is to construct a knot $t_{i}$ for each $P_{i}, 1 \leq i \leq n$. The constructed knots satisfy the following the condition: if the set of data points are taken from a parametric quadratic polynomial, i.e.,

$$
P_{i}=A \xi_{i}^{2}+B \xi_{i}+C, \quad 1 \leq i \leq n
$$

where $A=\left(a_{1}, a_{2}\right), B=\left(b_{1}, b_{2}\right)$ and $C=\left(c_{1}, c_{2}\right)$ are $2 \mathrm{D}$ points, then

$$
t_{i}-t_{i-1}=\alpha\left(\xi_{i}-\xi_{i-1}\right), \quad 1 \leq i \leq n
$$

for some constant $\alpha>0$.

Such a set of knots $t_{i}, 1 \leq i \leq n$, is known to have a quadratic polynomial precision. Obviously, using the knots satisfying equation (2), an interpolation scheme which reproduces quadratic polynomials will reproduce parametric quadratic polynomials.

Following, the basic idea in determining the knots $t_{i}, 1 \leq i \leq n$, will be described. If the set of data points is taken from a parametric quadratic polynomial, $P(\xi)=(x(\xi), y(\xi))$ defined by

$$
\begin{aligned}
& x(\xi)=X_{2} \xi^{2}+X_{1} \xi+X_{0}, \\
& y(\xi)=Y_{2} \xi^{2}+Y_{1} \xi+Y_{0},
\end{aligned}
$$

then, there is a rotating transformation to transform it to the following parabola form, as shown in Figure 1 .

$$
\begin{aligned}
& \bar{y}=a_{1} t^{2}+b_{1} t+c_{1}, \\
& \bar{x}=t
\end{aligned}
$$

Then the knots $t_{i}, 1 \leq i \leq n$ can be defined by

$$
t_{i}=\bar{x}_{i}, \quad i=1,2,3, \cdots, n,
$$

which has a quadratic polynomial precision. Assume that the following transformation

$$
\begin{aligned}
& \bar{x}=x \cos \beta_{2}+y \sin \beta_{2} \\
& \bar{y}=-x \sin \beta_{2}+y \cos \beta_{2}
\end{aligned}
$$

transforms $P(\xi)$ (3) to the parabola (4), then we have the following theorem 1 . 


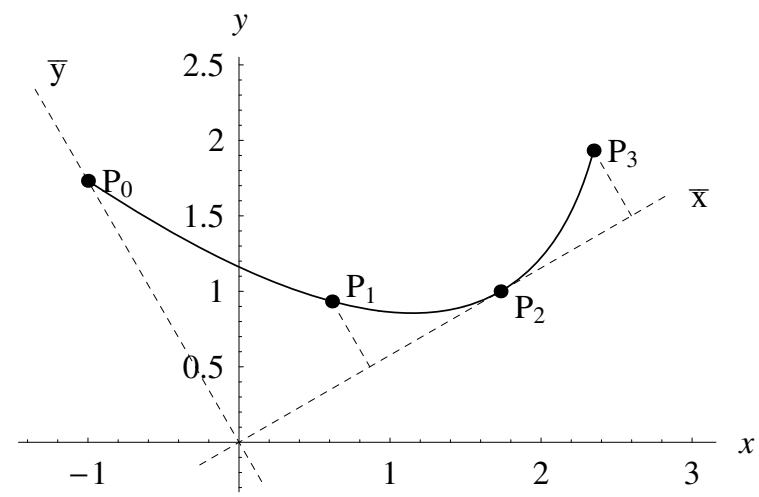

Fig. 1. A standard parabola in $\bar{x} \bar{y}$ coordinate system

Theorem 1. If the set of data points is taken from a parametric quadratic polynomial, $P(\xi)$ (3), then the knot $t_{i}, i=1,2,3, \cdots, n$ which have a quadratic polynomial precision, can be defined by

$$
\begin{aligned}
& t_{1}=0 \\
& t_{i}=t_{i-1}+\left(x_{i}-x_{i-1}\right) \cos \beta_{2}+\left(y_{i}-y_{i-1}\right) \sin \beta_{2}, \quad i=2,3, \cdots, n
\end{aligned}
$$

where

$$
\begin{aligned}
& \sin \beta_{2}=-X_{2} / \sqrt{X_{2}^{2}+Y_{2}^{2}} \\
& \cos \beta_{2}=Y_{2} /{\sqrt{X_{2}^{2}+Y_{2}^{2}}}^{2}
\end{aligned}
$$

Proof. In the $\bar{x} \bar{y}$ coordinate system, it follows from (3) that

$$
\begin{aligned}
& \bar{x}=\left(X_{2} \xi^{2}+X_{1} \xi+X_{0}\right) \cos \beta_{2}+\left(Y_{2} \xi^{2}+Y_{1} \xi+Y_{0}\right) \sin \beta_{2} \\
& \bar{y}=-\left(X_{2} \xi^{2}+X_{1} \xi+X_{0}\right) \sin \beta_{2}+\left(Y_{2} \xi^{2}+Y_{1} \xi+Y_{0}\right) \cos \beta_{2}
\end{aligned}
$$

If $\sin \beta_{2}$ and $\cos \beta_{2}$ are defined by (6), then the first expression of (7) becomes

$$
\xi=\frac{\bar{x}+X_{0} \cos \beta_{2}+Y_{0} \sin \beta_{2}}{X_{1} \cos \beta_{2}+Y_{1} \sin \beta_{2}}
$$

Substituting (8) into the second expression of (17) and rearranging, a parabola is obtained, which is defined by

$$
\bar{y}=a_{1} \bar{x}^{2}+b_{1} \bar{x}+c_{1},
$$

where $a_{1}, b_{1}$ and $c_{1}$ are defined by

$$
\begin{aligned}
& a_{1}=Y_{2} \cos \beta_{2}-X_{2} \sin \beta_{2} \\
& b_{1}=-2 a_{1} A B+\left(Y_{1} \cos \beta_{2}-X_{1} \sin \beta_{2}\right) A \\
& c_{1}=a_{1} A^{2} B^{2}-\left(Y_{1} \cos \beta_{2}-X_{1} \sin \beta_{2}\right) A B+Y_{0} \cos \beta_{2}-X_{0} \sin \beta_{2}
\end{aligned}
$$

Thus, $t_{i}$ can be defined by $\bar{x}_{i}$, i.e., the knot $t_{i}, i=1,2,3, \cdots, n$ can be defined by (5), which has a quadratic polynomial precision. 
The discussion above showed that the key point of determining knots is to construct the quadratic polynomial, $P(\xi)=(x(\xi), y(\xi))$ (3) using the given data points. This will be discussed in Section 3 .

\section{Determining Knots}

In this section, we first discuss how to construct a quadratic polynomial with four points, then discuss the determination of knots using the quadratic polynomial.

\subsection{Constructing a Quadratic Polynomial with Four Points}

Let $Q_{i}(\xi)$ be a parametric quadratic polynomial which interpolates $P_{i-1}, P_{i}$ and $P_{i+1} . Q_{i}(\xi)$ can be defined on the interval $[0,1]$ as follows:

$$
Q_{i}(s)=\psi_{1}(s)\left(P_{i-1}-P_{i}\right)+\psi_{2}(s)\left(P_{i+1}-P_{i}\right)+P_{i}
$$

where

$$
\begin{aligned}
& \psi_{1}(s)=\frac{\left(s-s_{i}\right)(s-1)}{s_{i}} \\
& \psi_{2}(s)=\frac{s\left(s-s_{i}\right)}{1-s_{i}}
\end{aligned}
$$

where $0<s_{i}<1$.

Expressions (9) and (10) show that four data points are needed to determine a parametric quadratic polynomial uniquely.

Let $P_{j}=\left(x_{j}, y_{j}\right), i-1 \leq j \leq i+2$, be four points in which there are no three points on a straight line. The $P_{i+2}$ will be used to determine $s_{i}$ (10) .

Without loss of generality, the coordinates of $P_{i-1}, P_{i}, P_{i+1}$ and $P_{i+2}$ are supposed to be $(0,1),(0,0),(1,0)$ and $\left(x_{i+2}, y_{i+2}\right)$, respectively, as shown in Figure 2. In the $x y$ coordinate system, $Q_{i}(s)$ defined by (9) becomes

$$
\begin{aligned}
& x=s\left(s-s_{i}\right) /\left(1-s_{i}\right) \\
& y=\left(s-s_{i}\right)(s-1) / s_{i}
\end{aligned}
$$

Let $s_{i+2}$ be the knot associated with the point $\left(x_{i+2}, y_{i+2}\right)$. As point $\left(x_{i+2}, y_{i+2}\right)$ is on the curve, we have

$$
\begin{aligned}
& x_{i+2}=s_{i+2}\left(s_{i+2}-s_{i}\right) /\left(1-s_{i}\right) \\
& y_{i+2}=\left(s_{i+2}-s_{i}\right)\left(s_{i+2}-1\right) / s_{i}
\end{aligned}
$$

It follows from (12) that

$$
s_{i+2}=x_{i+2}+\left(1-x_{i+2}-y_{i+2}\right) s_{i}
$$

Substituting (13) into (12), one gets the following equation:

$$
s_{i}^{2}+A\left(x_{i+2}, y_{i+2}\right) s_{i}+B\left(x_{i+2}, y_{i+2}\right)=0
$$




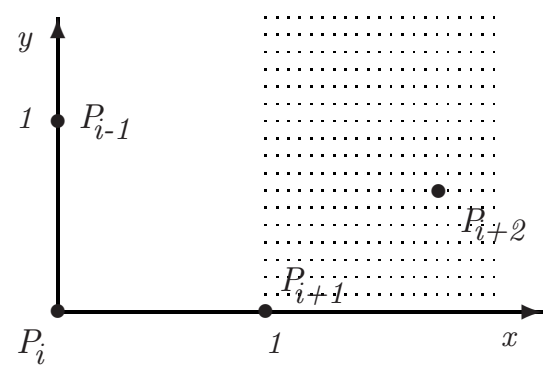

Fig. 2. $P_{i+2}$ is in the dotted region

where

$$
\begin{aligned}
& A\left(x_{i+2}, y_{i+2}\right)=-\frac{2 x_{i+2}}{x_{i+2}+y_{i+2}} \\
& B\left(x_{i+2}, y_{i+2}\right)=\frac{\left(1-x_{i+2}\right) x_{i+2}}{\left(1-x_{i+2}-y_{i+2}\right)\left(x_{i+2}+y_{i+2}\right)}
\end{aligned}
$$

As $s_{i+2}>1$, the root of (14) is

$$
s_{i}=\frac{1}{x_{i+2}+y_{i+2}}\left(x_{i+2}+\sqrt{\frac{x_{i+2} y_{i+2}}{x_{i+2}+y_{i+2}-1}}\right)
$$

It follows from (9) - (10) that if the given data points are taken from a parametric quadratic polynomial $Q(t)$, then there is an unique $s_{i}$ satisfying $0<s_{i}<1$ to make the curve $Q_{i}(s)$ (9) pass through the given data points. Since $s_{i}$ is determined uniquely by (15), $Q_{i}(s)$ is equivalent to $Q(t)$.

Substituting $s_{i+2}>1$ into (11) one obtains

$$
x_{i+2}>1 \text { and } y_{i+2}>0,
$$

that is, the point $\left(x_{i+2}, y_{i+2}\right)$ should be on the dotted region in Figure 2.

\subsection{Determining Knots}

After $s_{i}$ being determined, $Q_{i}(s)$ (9) can be written as

$$
\begin{aligned}
& x_{i}(s)=X_{i, 2} s^{2}+X_{i, 1} s+X_{i, 0}, \\
& y_{i}(s)=Y_{i, 2} s^{2}+Y_{i, 1} s+Y_{i, 0},
\end{aligned}
$$

where

$$
\begin{aligned}
& X_{i, 2}=\frac{x_{i-1}-x_{i}}{s_{i}}+\frac{x_{i+1}-x_{i}}{1-s_{i}} \\
& X_{i, 1}=-\frac{\left(x_{i-1}-x_{i}\right)\left(s_{i}+1\right)}{s_{i}}-\frac{\left(x_{i+1}-x_{i}\right) s_{i}}{1-s_{i}} \\
& X_{i, 0}=x_{i-1} \\
& Y_{i, 2}=\frac{y_{i-1}-y_{i}}{s_{i}}+\frac{y_{i+1}-y_{i}}{1-s_{i}} \\
& Y_{i, 1}=-\frac{\left(y_{i-1}-y_{i}\right)\left(s_{i}+1\right)}{s_{i}}-\frac{\left(y_{i+1}-y_{i}\right) s_{i}}{1-s_{i}} \\
& Y_{i, 0}=y_{i-1}
\end{aligned}
$$


It follows from Theorem 1 that for $i=2,3, \cdots, n-2$, the knot interval $t_{j+1}-t_{j}=$ $\Delta_{j}^{i}$ between $P_{j}$ and $P_{j+1}, j=i-1, i, i+1$ can be defined by

$$
\Delta_{j}^{i}=\left(x_{j+1}-x_{j}\right) \cos \beta_{i}+\left(y_{j+1}-y_{j}\right) \sin \beta_{i}, \quad j=i-1, i, i+1
$$

where $\cos \beta_{i}$ and $\sin \beta_{i}$ are defined by (please see (6) $)$

$$
\begin{aligned}
& \sin \beta_{i}=-X_{i, 2} / \sqrt{X_{i, 2}^{2}+Y_{i, 2}^{2}} \\
& \cos \beta_{i}=Y_{i, 2} / \sqrt{X_{i, 2}^{2}+Y_{i, 2}^{2}}
\end{aligned}
$$

Based on the definition (19) that for the pair of $P_{1}$ and $P_{2}$, there is one knot interval $\Delta_{1}^{2}$; for the pair of $P_{2}$ and $P_{3}$, there are two knot intervals, $\Delta_{2}^{2}$ and $\Delta_{2}^{3}$; for the pair of $P_{i}$ and $P_{i+1}, 3 \leq i \leq n-2$, there are three knot intervals, $\Delta_{i}^{i-1}$, $\Delta_{i}^{i}$ and $\Delta_{i}^{i+1}$; the knot intervals for the pair of $P_{j-1}$ and $P_{j}, j=n-1, n$ are similar. Now the knot interval $\Delta_{i}$ for the pair of $P_{i}$ and $P_{i+1}, i=1,3, \cdots, n-1$ are defined by

$$
\begin{aligned}
& \Delta_{1}=\Delta_{1}^{2} \\
& \Delta_{2}=\Delta_{2}^{2}+\delta_{2}^{2} \\
& \Delta_{i}=\Delta_{i}^{i}+2 \delta_{i}^{1} \delta_{i}^{2} /\left(\delta_{i}^{1}+\delta_{i}^{2}\right), \quad i=3,4, \cdots, n-3 \\
& \Delta_{n-2}=\Delta_{n-2}^{n-2}+\delta_{n-2}^{1} \\
& \Delta_{n-1}=\Delta_{n-1}^{n-2}
\end{aligned}
$$

where

$$
\begin{aligned}
& \delta_{i}^{1}=\left|\Delta_{i}^{i}-\Delta_{i}^{i-1}\right| \\
& \delta_{i}^{2}=\left|\Delta_{i}^{i}-\Delta_{i}^{i+1}\right|
\end{aligned}
$$

If the given set of data points are taken from a parametric quadratic polynomial, then $\delta_{i}^{1}=\delta_{i}^{2}=0$. The terms $\delta_{2}^{2}, 2 \delta_{i-1}^{1} \delta_{i-1}^{2} /\left(\delta_{i-1}^{1}+\delta_{i-1}^{2}\right)$ and $\delta_{n-2}^{1}$ are correction to $\Delta_{2}^{2}, \Delta_{i}^{i}, i=4,5, \cdots, n-2$ and $\Delta_{n-2}^{n-2}$, respectively.

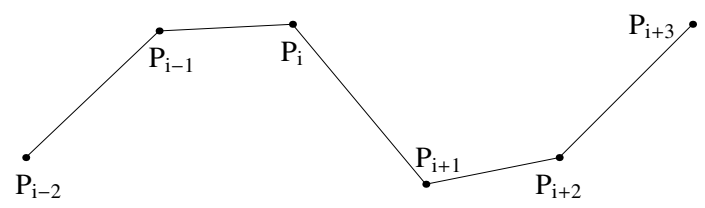

Fig. 3. Example 1 of the data points

For the data points as shown in Figure 3, as the data points change its convexity, the knot interval between $P_{i}$ and $P_{i+1}$ is defined by the combination of $\Delta_{i-1}^{i}$ and $\Delta_{i+1}^{i}$, i.e, by

$$
\Delta_{i}=\left(\Delta_{i-1}^{i}+\Delta_{i+1}^{i}\right) / 2
$$

While for data points as shown in Figure 4 the knot intervals are determined by subdividing the data points at point $P_{i+1}$ into two sets of data points. The first 


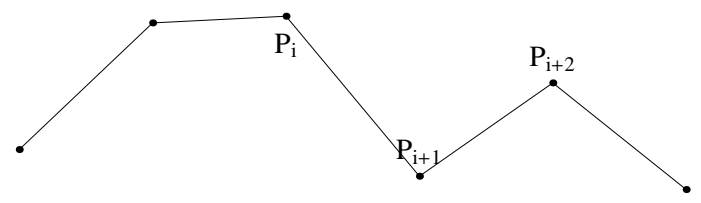

Fig. 4. Example 2 of the data points

set of data point ends at $P_{i+1}$, while the second set of data point starts at $P_{i+1}$. If $P_{i-1}, P_{i}$ and $P_{i+1}$ are on a straight line, then setting $t_{i}-t_{i-1}=\left|P_{i-1} P_{i}\right|$, $t_{i+1}-t_{i}=\left|P_{i} P_{i+1}\right|$, this determination makes the quadratic polynomial $Q_{i}(t)$ which passes $P_{i-1}, P_{i}$ and $P_{i+1}$ be a straight line with the magnitude of the first derivative being a constant. Such a straight line is the most well defined curve one can get in this case.

\section{Experiments}

The new method has been compared with the chord length, centripetal, Foley and ZCM's methods. The comparison is performed by using the knots computed using these methods in the construction of a parametric cubic spline interpolant. For brevity, the cubic splines produced using these methods are called chord spline, centripetal spline, Foley's spline, ZCM spline and new spline, respectively. The data points used in the comparison are taken from the following ellipse

$$
\begin{aligned}
& x=x(\tau)=3 \cos (2 \pi \tau) \\
& y=y(\tau)=2 \sin (2 \pi \tau)
\end{aligned}
$$

The comparison is performed by dividing the interval $[0,1]$ into 36 sub-intervals to define data points, i.e., $\tau_{i}$ is defined by

$$
\tau_{i}=(i+\sigma \sin ((36-i) * i)) / 36 \quad i=0,1,2, \cdots, 36
$$

where $0 \leq \sigma \leq 0.25$.

To avoid the maximum error occurred near the end points $\left(x_{0}, y_{0}\right)$ and $\left(x_{20}\right.$, $\left.y_{20}\right)$, the tangent vectors of $F(\tau)$ at $\tau=0$ and $\tau=1$ are used as the end conditions to construct the cubic splines.

The five methods are compared by the absolute error curve $E(t)$, defined by

$$
\begin{aligned}
E(t) & =\min \{|P(t)-F(\tau)|\} \\
& =\min \left\{\left|P_{i}(t)-F(\tau)\right|, \tau_{i} \leq \tau \leq \tau_{i+1}\right\} \quad i=0,1,2, \cdots, 19
\end{aligned}
$$

where $P(t)$ denotes one of the chord spline, centripetal spline, Foley's spline, ZCM's spline or new spline, $P_{i}(t)$ is the corresponding part of $P(t)$ on the subinterval $\left[t_{i}, t_{i+1}\right]$, and $F(\tau)$ is defined by (22). For the point $P(t), E(t)$ is the shortest distance from curve $F(\tau)$ to $P(t)$. 
Table 1. Maximum absolute errors

\begin{tabular}{|c|c|c|c|c|c|}
\hline Error & chord & centripetal & Foley & ZCM & New \\
\hline$\sigma=.0$ & $5.29 \mathrm{e}-5$ & $5.29 \mathrm{e}-5$ & $5.29 \mathrm{e}-5$ & $5.29 \mathrm{e}-5$ & $5.29 \mathrm{e}-5$ \\
\hline$\sigma=.05$ & $1.67 \mathrm{e}-4$ & $3.71 \mathrm{e}-3$ & $2.39 \mathrm{e}-3$ & $1.58 \mathrm{e}-4$ & $1.60 \mathrm{e}-4$ \\
\hline$\sigma=.10$ & $3.17 \mathrm{e}-4$ & $8.00 \mathrm{e}-3$ & $5.33 \mathrm{e}-3$ & $2.93 \mathrm{e}-4$ & $2.89 \mathrm{e}-4$ \\
\hline$\sigma=.15$ & $5.08 \mathrm{e}-4$ & $1.30 \mathrm{e}-2$ & $8.88 \mathrm{e}-3$ & $4.58 \mathrm{e}-4$ & $4.37 \mathrm{e}-4$ \\
\hline$\sigma=.20$ & $7.41 \mathrm{e}-4$ & $1.86 \mathrm{e}-2$ & $1.31 \mathrm{e}-2$ & $6.55 \mathrm{e}-4$ & $6.04 \mathrm{e}-4$ \\
\hline$\sigma=.25$ & $1.02 \mathrm{e}-3$ & $2.49 \mathrm{e}-2$ & $1.79 \mathrm{e}-2$ & $8.86 \mathrm{e}-4$ & $7.88 \mathrm{e}-4$ \\
\hline
\end{tabular}

The maximum values of the error curve $E(t)$ generated by these methods are shown in table 1. The five methods have also been compared on data points which divide $[0,1]$ into $18,72, \ldots$ etc subintervals. The results are basically similar as those shown in tables 1 .

\section{Conclusions and Future Works}

A new method for determining knots in parametric curve interpolation is presented. The determined knots have a quadratic polynomial precision. This means that from the approximation point of view, the new method is better than the chord length, centripetal and Foley's methods in terms of error evaluation in the associated Taylor series. The ZCM's method has also a quadratic polynomial precision, but it is a global method, while the new method is a local one.

The new method works well on the data points whose convexity does not change sign, our next work is to extend it to work on the data points whose convexity changes sign.

Acknowledgments. This work was supposed by the National Key Basic Research 973 program of China(2006CB303102), the National Natural Science Foundation of China(60533060, 60633030).

\section{References}

1. Ahlberg, J. H., Nilson, E. N. and Walsh, J. L., The theory of splines and their applications, Academic Press, New York, NY, USA, 1967.

2. de Boor, C., A practical guide to splines, Springer Verlag, New York, 1978.

3. Lee, E. T. Y., Choosing nodes in parametric curve interpolation, CAD, Vol. 21, No. 6, pp. 363-370, 1989.

4. Farin G., Curves and surfaces for computer aided geometric design: A practical guide, Academic Press, 1988.

5. Zhang, C., Cheng, F. and Miura, K., A method for determing knots in parametric curve interpolation, CAGD 15(1998), pp 399-416. 